\title{
Chronic Kidney Disease Is an Independent Predictor of Adverse Clinical Outcomes in Patients with Recent Small Subcortical Infarcts
}

\author{
Naoki Saji Takahiro Sato Kenichi Sakuta Junya Aoki Kazuto Kobayashi \\ Noriko Matsumoto Junichi Uemura Kensaku Shibazaki Kazumi Kimura
}

Department of Stroke Medicine, Kawasaki Medical School, Kurashiki, Japan

Key Words

Chronic kidney disease - Lacunar stroke - Renal impairment - Recent small subcortical infarcts $\cdot$ Small vessel disease $\cdot$ Stroke

\section{Abstract}

Background: Chronic kidney disease (CKD) is associated with cerebral small vessel diseases (SVD) and predicts stroke, cardiovascular events and mortality. However, its association with recent small subcortical infarcts (RSSI), a novel subtype of cerebral SVD, has not yet been established in stroke patients. The aim of this longitudinal study was to clarify whether CKD can predict clinical outcome in patients with RSSI. Methods: We enrolled patients with first-ever RSSI (formerly categorized as acute lacunar stroke). CKD was defined as an estimated glomerular filtration rate of $<60 \mathrm{ml} / \mathrm{min} / 1.73 \mathrm{~m}^{2}$ on admission. The patients were divided into two groups according to the presence or absence of CKD. The endpoints were recurrent stroke, cardiovascular events or all-cause mortality. The patients were followed up at 3, 6 and 12 months after stroke onset and yearly thereafter. Event-free survival analysis was undertaken using Kaplan-Meier plots and the log-rank test. Cox's proportional-hazards analysis was conducted regarding age, sex and the presence of any cerebral SVD. Results: A total of 152 patients (66\% males; mean age: 67.6 years) were consecutively enrolled, and 44 (29\%) had CKD. During the follow-up period (median: 3 years; interquartile range: 1-4), 27 patients (18\%) reached endpoints. The numbers of patients per endpoint were as follows: all-cause mortality 14, ischemic stroke 9, hemorrhagic stroke 2 and aortic dissection 2. Patients with CKD were significantly older (77 vs. 64 years; $p<0.001)$, had higher serum creatinine $(0.96$ vs. 0.65 mg/ 
Saji et al.: Chronic Kidney Disease Is an Independent Predictor of Adverse Clinical Outcomes in Patients with Recent Small Subcortical Infarcts

$\mathrm{dl} ; \mathrm{p}<0.001$ ), higher brain natriuretic peptide (51.1 vs. $18.5 \mathrm{pg} / \mathrm{ml} ; \mathrm{p}<0.001$ ) and a higher National Institutes of Health Stroke Scale score on admission ( 3 vs. $2 ; p<0.001$ ), and were less likely to have modified Rankin Scale scores of $0-2$ after stroke onset (52 vs. $77 \% ; p=0.003$ ). Patients with white matter hyperintensity [odds ratio (OR) 3.0; 95\% confidence interval (CI): $1.5-6.2 ; p=0.003$ ] and those with microbleeds (OR 2.5; 95\% CI: $1.2-5.1 ; p=0.015$ ) had more pronounced CKD than the remaining patients. A Kaplan-Meier curve analysis showed that patients with CKD had a less favorable outcome than those without CKD $(p<0.001)$. The multivariate Cox proportional-hazards analysis revealed that CKD was associated with recurrent stroke, cardiovascular events or all-cause mortality (hazard ratio 2.22; 95\% CI: 1.12-4.25; $\mathrm{p}=$ 0.02). Conclusions: CKD was found to be independently associated with recurrent stroke, cardiovascular events or all-cause mortality in patients with RSSI.

(c) 2014 S. Karger AG, Basel

\section{Introduction}

Chronic kidney disease (CKD) is now an accepted risk factor for cardiovascular diseases, stroke and dementia and is a surrogate marker for adverse clinical outcomes in patients with stroke [1-7]. Patients with CKD are known to have endothelial dysfunction and are at risk from both thrombotic and hemorrhagic events [5-10]. Furthermore, CKD is associated with cerebral small vessel diseases (SVD) [2, 9-13] because they share similar anatomical and physiologic characteristics.

Cerebral SVD are hypertension-related cerebral changes and are focused on as a risk factor for stroke [14]. Recently, cerebral SVD have been reclassified into five categories according to the Standards for Reporting Vascular Changes on Neuroimaging (STRIVE) recommendations as follows: recent small subcortical infarcts (RSSI; formerly categorized as acute lacunar stroke); white matter hyperintensity (WMH); lacunae (formerly defined as silent lacunar infarcts); microbleeds, and perivascular space [14]. Although CKD is closely associated with cerebral SVD, the association between CKD and clinical outcomes in patients with RSSI has not yet been clarified. Several studies have assessed CKD in cohorts of patients with stroke $[3,4,6,7,15]$, in cohorts of patients with ischemic stroke $[5,9,11]$ and in the general population $[1,2,10,12,13,16]$. Moreover, it is unclear as to whether or not CKD affects the longitudinal clinical outcomes of patients with RSSI.

The aim of the present study was to clarify the prognosis of patients with RSSI, and, in particular, to determine whether CKD per se could affect adverse outcomes in such patients. We hypothesized that CKD is associated with poor outcome in patients with RSSI independent of the presence of cerebral SVD.

\section{Subjects and Methods}

\section{Study Design}

Between April 2007 and March 2013, we enrolled consecutive patients with first-ever RSSI who were admitted to the Kawasaki Medical School Hospital within $24 \mathrm{~h}$ of stroke onset. An eligible infarct size was defined as $<15 \mathrm{~mm}$ in diameter detected on diffusion-weighted imaging (DWI) scans on admission according to the Trial of Org 10172 in Acute Stroke Treatment (TOAST) criteria [17] and the STRIVE recommendations [14]. We excluded patients with recurrent stroke. This longitudinal observational study complied with the Declaration of Helsinki and was approved by the Institutional Review Board at the Kawasaki Medical School Hospital. Informed consent was obtained from all patients. This trial is 
Saji et al.: Chronic Kidney Disease Is an Independent Predictor of Adverse Clinical Outcomes in Patients with Recent Small Subcortical Infarcts

registered with the UMIN Clinical Trials Registry (UMIN000013767). Detailed information is provided in the online supplementary file (for all online suppl. material, see www. karger.com/doi/10.1159/000365565).

\section{Baseline Assessment}

We assessed age, sex, body mass index and blood pressure on admission, and known vascular risk factors such as hypertension, dyslipidemia, diabetes mellitus, ischemic heart disease and current smoking habits. Serum creatinine was measured on admission, and the estimated glomerular filtration rate (eGFR) was determined using the equation proposed by the Japanese Society of Nephrology as follows: eGFR $\left(\mathrm{ml} / \mathrm{min} / 1.73 \mathrm{~m}^{2}\right)=194 \times$ [serum creatinine $(\mathrm{mg} / \mathrm{dl})]^{-1.094} \times$ [age (years) $]^{-0.287} \times 0.739$ (if female) [5]. CKD was defined as an eGFR of $<60 \mathrm{ml} / \mathrm{min} / 1.73 \mathrm{~m}^{2}$ on admission $[2,4,11]$. Stroke subtype was classified according to the TOAST criteria [17]. Stroke neurologists assessed the National Institutes of Health Stroke Scale (NIHSS) scores on admission and at discharge. The modified Rankin Scale (mRS) scores at 90 days were assessed. We defined mRS scores of $0-2$ as a good outcome and scores of 3-5 as a poor outcome [18].

\section{Magnetic Resonance Imaging}

The patients underwent a 1.5-tesla MRI of the brain (Signa EXCITE XL version 11.0; GE Healthcare, Milwaukee, Wis., USA), including DWI, fluid-attenuated inversion recovery (FLAIR) imaging, T2*-weighted gradient echo imaging (T2*) and 3D time-of-flight MR angiography. The neuroimaging protocol is provided in the online supplementary file. RSSI was defined as infarcts of $<15 \mathrm{~mm}$ in diameter as detected using DWI scans on admission [14]. Lacunae, formerly categorized as silent lacunar infarcts, were defined as a focal lesion of $\geq 3$ $\mathrm{mm}$ in diameter, with hyperintensity on T2WI and hypointensity on FLAIR images [18-22]. WMH was defined as an irregular periventricular hyperintensity (Fazekas grade $\geq 3$ ) and/or early confluent or confluent separate deep white matter hyperintense lesions (Fazekas grade $\geq 2$ ) on T2WI and FLAIR images [18-22]. Microbleeds were defined as a focal area of signal loss in the brain parenchyma of a size of $<5 \mathrm{~mm}$ on T2* scans [14]. A trained neurologist (N.S.) assessed all scans while blinded to the patients' details.

\section{Follow-Up}

The patients were followed up at 3, 6 and 12 months after stroke onset and yearly thereafter. Trained research assistants who were blinded to the clinical data assessed the patients by telephone interview using a standardized interview form. The follow-up ended on 31 May, 2014.

\section{Endpoints}

The present study's endpoints were recurrent stroke, cardiovascular events (defined as myocardial infarction, heart failure and arterial dissection) or all-cause mortality. Patients with such status were thoroughly assessed using multiple concurrent approaches including a review of hospital and outpatient medical records and computerized databases and, if necessary, by contacting the patients, their families or their primary care physician.

\section{Statistical Analysis}

Continuous variables, ordinal variables and categorical variables were compared using the unpaired Student $t$ test, the Wilcoxon rank-sum test and the $\chi^{2}$ test, respectively. First, we divided the patients into two groups according to the presence or absence of CKD to compare their clinical characteristics (table 1). Second, we compared the frequency of CKD in patients with lacunae, WMH and microbleeds and patients who did not have these conditions (fig. 1). 


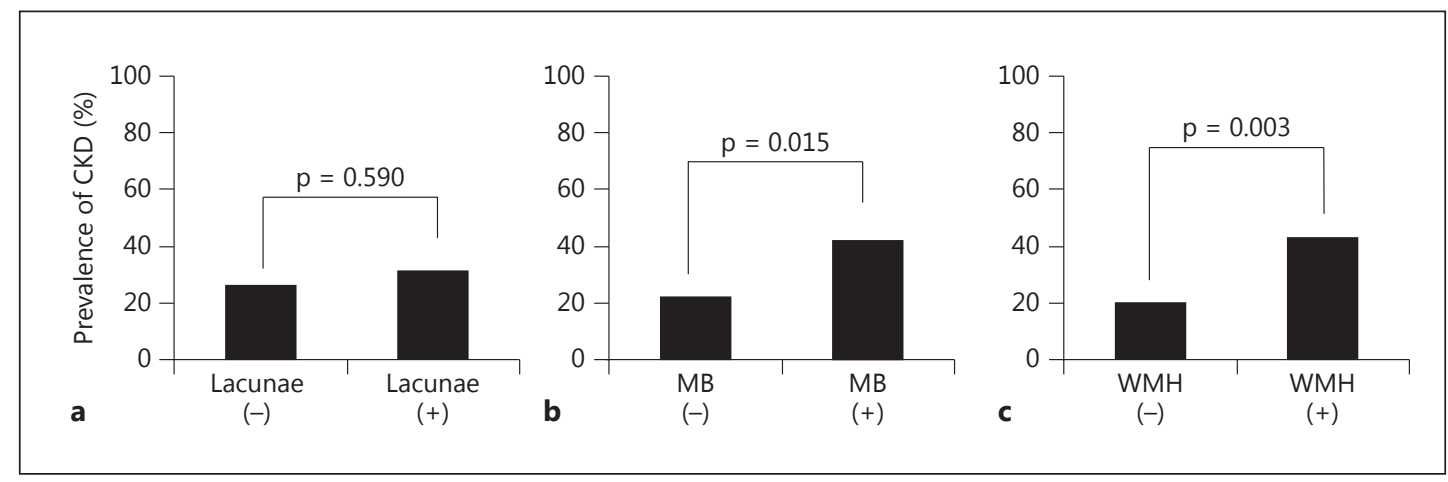

Fig. 1. Association between CKD and lacunae, microbleeds (MB) and WMH. Prevalence of CKD in patients according to the presence $(+)$ or absence $(-)$ of lacunae $(\mathbf{a}), \mathrm{MB}(\mathbf{b})$ and WMH (c).

Table 1. Characteristics of the patients with and without CKD

\begin{tabular}{lccr}
\hline & $\begin{array}{l}\text { With CKD } \\
(\mathrm{n}=44 ; 29 \%)\end{array}$ & $\begin{array}{l}\text { Without CKD } \\
(\mathrm{n}=108 ; 71 \%)\end{array}$ & p value \\
\hline Male & $29(66)$ & $72(67)$ & 1.000 \\
Age, years & $77(70-82)$ & $64(59-71)$ & $<0.001$ \\
Serum creatinine, mg/dl & $0.96(0.70-1.22)$ & $0.65(0.53-0.74)$ & $<0.001$ \\
BNP, pg/ml & $51.1(21-85.2)$ & $18.5(10.2-31.3)$ & $<0.001$ \\
eGFR, ml/min/1.73 m ${ }^{2}$ & $47.2(30.9-55.0)$ & $85.2(73.9-102.5)$ & $<0.001$ \\
Edaravone, mg & $180(0-360)$ & $330(188-413)$ & 0.026 \\
SBP, mm Hg & $160(141-178)$ & $164(156-188)$ & 0.110 \\
DBP, mm Hg & $86(76-98)$ & $92(80-106)$ & 0.049 \\
BMI & $21.4(19.1-24.5)$ & $23.7(22.4-26.0)$ & 0.001 \\
NIHSS score on admission & $3(2-5)$ & $2(1-4)$ & 0.038 \\
NIHSS score at discharge & $1(0-3)$ & $1(0-2)$ & 0.673 \\
mRS score of 0-2 at discharge & $23(52)$ & $84(77)$ & 0.003 \\
WMH & $25(57)$ & $33(31)$ & 0.003 \\
Lacunae & $26(59)$ & $57(53)$ & 0.590 \\
Microbleeds & $22(50)$ & $31(29)$ & 0.015 \\
Hypertension & $32(73)$ & $77(71)$ & 1.000 \\
Diabetes mellitus & $17(39)$ & $28(26)$ & 0.170 \\
Dyslipidemia & $16(36)$ & $29(27)$ & 0.248 \\
Ischemic heart disease & $1(3)$ & $4(4)$ & 1.000 \\
Smoking & $18(41)$ & $56(52)$ & 0.283 \\
Alcohol consumption & $15(34)$ & $11(10)$ & 0.465 \\
Endpoint & $16(36)$ & $<0.001$ \\
\hline
\end{tabular}

Data represent medians with interquartile ranges in parentheses or numbers of patients with percentages in parentheses. Endpoints were recurrent stroke, cardiovascular events and all-cause mortality. BNP = Brain natriuretic peptide; SBP = systolic blood pressure; $\mathrm{DBP}=$ diastolic blood pressure; $\mathrm{BMI}=$ body mass index.

Third, event-free survival analysis was undertaken using Kaplan-Meier plots and the log-rank test to compare patients with CKD and those without CKD (fig. 2). Fourth, the Cox proportional-hazards model (table 2) was used to estimate the risk of recurrent stroke, cardiovascular events or all-cause mortality associated with CKD (presence or absence), unadjusted, adjusted for age and sex (model 1) and adjusted for age, sex and the presence of cerebral SVD (presence of any lacunae, WMH or microbleeds; model 2). Odds ratios (OR) and hazard ratios 


\section{Cerebrovascular \\ Diseases}

Fig. 2. Kaplan-Meier survival curve estimates of the risk of recurrent stroke, cardiovascular events or all-cause mortality associated with CKD.

Table 2. Association between CKD and the endpoints evaluated using the Cox proportionalhazards model

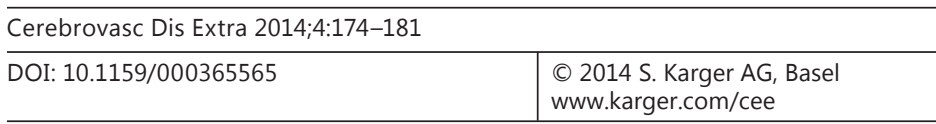

Saji et al.: Chronic Kidney Disease Is an Independent Predictor of Adverse Clinical Outcomes in Patients with Recent Small Subcortical Infarcts

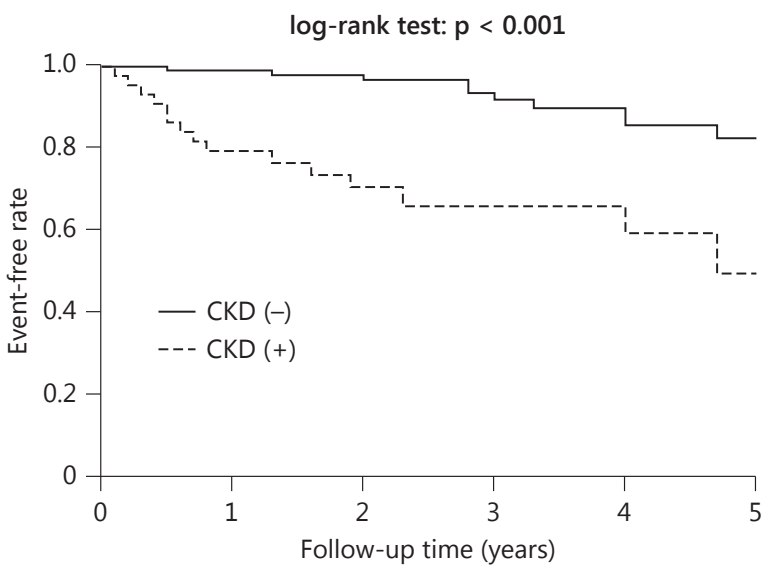

Patients at risk, $\mathrm{n}$

$\begin{array}{lllllll}\text { CKD (-) } & 108 & 103 & 82 & 55 & 41 & 26\end{array}$

$\begin{array}{lllllll}C K D(+) & 44 & 35 & 24 & 14 & 10 & 5\end{array}$

\begin{tabular}{llll}
\hline & HR & $95 \% \mathrm{CI}$ & $\mathrm{p}$ value \\
\hline Unadjusted & 2.56 & $1.42-4.49$ & 0.002 \\
Model 1 & 2.33 & $1.19-4.44$ & 0.015 \\
Model 2 $^{\mathrm{b}}$ & 2.22 & $1.12-4.25$ & 0.023 \\
\hline
\end{tabular}

Endpoints were recurrent stroke, cardiovascular events and all-cause mortality. $\mathrm{HR}=$ Hazard ratio.

${ }^{\text {a }}$ Adjusted for age and sex.

${ }^{\mathrm{b}}$ Adjusted for age, sex and the presence of any lacunae, WMH or microbleeds.

are presented along with 95\% confidence intervals (CI). All comparisons were two-tailed, and a p value $<0.05$ was considered being statistically significant. All data were analyzed using the JMP 11.0 software package (SAS Institute Inc., Cary, N.C., USA). Detailed information is provided in the online supplementary file.

\section{Results}

\section{Patient Characteristics}

A total of 152 patients (66\% males; mean age: $67.6 \pm 11.1$ years) with first-ever RSSI, formerly defined as small vessel occlusion based on the TOAST criteria, were enrolled in the present study. Among these, 44 (29\%) had CKD. During the follow-up period, 27 patients (18\%) reached endpoints. The median follow-up time of the patients was 3 years (interquartile range: $1-4)$. The numbers of patients per endpoint were as follows: all-cause mortality 14, ischemic stroke 9, hemorrhagic stroke 2 and aortic dissection 2. 
Saji et al.: Chronic Kidney Disease Is an Independent Predictor of Adverse Clinica Outcomes in Patients with Recent Small Subcortical Infarcts

We compared characteristics between female and male patients. Female patients were significantly older ( 72 vs. 66 years; $p=0.01$ ), had a slightly higher NIHSS score on admission ( 3 vs. $2 ; \mathrm{p}=0.267$ ) and were less likely to have mRS scores of $0-2$ after stroke onset (59 vs. 76\%; OR 2.25; 95\% CI: 1.09-4.62; p < 0.05). There was no significant difference in the proportion of patients with CKD between females and males ( 29 vs. $29 \%$; $p=1.000$ ).

\section{Comparison of Patients with and without CKD}

Table 1 shows the comparison between patients with and without CKD. Patients with CKD were significantly older, had higher serum creatinine and brain natriuretic peptide levels as well as a higher NIHSS score on admission; they were less likely to have mRS scores of 0-2 after stroke onset, had a high frequency of WMH and microbleeds as well as a high possibility of all-cause mortality or recurrent stroke as compared with those without CKD.

\section{Association between CKD and Cerebral SVD}

The eGFR was significantly lower in patients with WMH (63.8 vs. $81.7 \mathrm{ml} / \mathrm{min} / 1.73 \mathrm{~m}^{2}$; $\mathrm{p}<0.001$ ) and in those with microbleeds ( $66.0 \mathrm{vs} .79 .0 \mathrm{ml} / \mathrm{min} / 1.73 \mathrm{~m}^{2} ; \mathrm{p}<0.01$ ) as compared with those who did not have these conditions; however, there was no significant difference between patients with lacunae and those without lacunae $\left(73.8 \mathrm{vs} .77 .3 \mathrm{ml} / \mathrm{min} / 1.73 \mathrm{~m}^{2} ; \mathrm{p}=\right.$ 0.197). Likewise, patients with WMH (OR 3.0; 95\% CI: 1.5-6.2; $\mathrm{p}=0.003$ ) and those with microbleeds (OR 2.5; 95\% CI: $1.2-5.1 ; \mathrm{p}=0.015$ ) had more CKD than the rest of the patients; however, there was no significant difference between patients with lacunae and those without lacunae (OR 1.3; 95\% CI: 0.6-2.6; $p=0.590$; fig. 1).

\section{Longitudinal Analysis}

A Kaplan-Meier curve analysis showed that patients with CKD had less favorable survival times free of recurrent stroke, cardiovascular events or all-cause mortality than those without CKD (log-rank test, $\mathrm{p}<0.001$; fig. 2). The multivariate Cox proportional-hazards model revealed that CKD was an independent predictor of recurrent stroke or all-cause mortality after adjusting for age, sex and the presence of any cerebral SVD (table 2).

\section{Discussion}

The main finding of the present study was that CKD was independently associated with recurrent stroke, cardiovascular events or all-cause mortality in patients with RSSI. Additionally, we confirmed a positive relationship between WMH, microbleeds and CKD.

In the general population, the prognosis of patients with CKD is poor as compared with those who do not have CKD [1]. Likewise, stroke patients with CKD have a severe neurological deficit, poor functional outcomes, a higher rate of long-term mortality and increased cardiovascular morbidity relative to stroke patients who do not have CKD $[4-7,11]$. However, such an association had not previously been confirmed in patients with RSSI. Furthermore, although there was a clear relationship between CKD and cerebral SVD in several earlier studies $[7,9-13,15,16]$, similar relationships in strictly selected stroke patients, in particular patients with RSSI, have not previously been assessed. Although we applied a combined endpoint (recurrent stroke, cardiovascular events and all-cause mortality) in the present study, RSSI patients with CKD had a significantly poorer outcome than CKD patients without RSSI. Our findings are in line with these previous studies and could provide a better understanding of both the association between small artery disease in the brain and kidney and the prognosis of stroke patients with RSSI. 
We also found a robust association between WMH (OR 3.0), microbleeds (OR 2.5) and CKD. This positive association has been previously reported in patients with stroke $[7,9,11$, $23]$ and in the general population [10,12,13]. As for lacunae, previous studies have indicated a significant association between lacunae (silent lacunar infarcts) and CKD [12, 16]. In contrast, no significant association between lacunae and CKD was found in the present study. We speculated that an equal background regarding the presence of lacunae in the patients who were enrolled in the present study could have led to lost statistical difference because our cohort consisted of patients with RSSI.

Similar mechanisms appear to be involved in the development of cerebral SVD and CKD. First, both cerebral SVD and CKD are mainly caused by hypertension-related small artery disease [8]. Second, similarities in hemodynamics between the vascular beds of the brain and kidney could explain this close association [2, 7-9]. Blood vessels in the brain and kidney are vulnerable to fluctuations in blood pressure [8]. Third, endothelial dysfunction underlies the development of both cerebral SVD and CKD $[8,11]$. Cerebral endothelial dysfunction could cause blood-brain barrier failure and lead to damage to the cerebral parenchyma [24]. Likewise, glomerular endothelial dysfunction could cause capillary vasculopathy and lead to an impairment in renal function [8]. Fourth, increased blood pressure caused by CKD might be responsible for the cerebral SVD [11]. Sodium retention, an activated renin-angiotensin system and elevated plasma catecholamine levels could increase blood pressure [11]. These combined factors might increase the risk of recurrent stroke, cardiovascular events or allcause mortality in the present study.

The strength of our study lies in the evaluation of a strictly selected group of patients with first-ever RSSI. The eligible infarct diameter was limited to $<15 \mathrm{~mm}$ as assessed by means of DWI scans on admission within $24 \mathrm{~h}$ of stroke onset in accordance with the TOAST criteria [17] and the STRIVE recommendations [14]. Although associations between cardioembolic stroke, atrial fibrillation and CKD have been reported [3], the confirmation of CKD as a clinical predictor in patients with cerebral SVD could provide a clear correlation between small artery diseases of the brain and the kidneys.

There are several limitations to our study. There is the possibility of selection bias, since this study was performed in a single hospital-based cohort. The number of patients included in the study was small. A combined endpoint was assessed in the present study. Other confounding factors such as albuminuria [8, 16], proteinuria [5] or arterial stiffness [19-22] were not assessed. Arterial stiffness can be used to assess the presence of cerebral SVD [19$22]$ and predicts cardiovascular events or all-cause mortality [25, 26]. Further research taking these factors into consideration will be needed to validate our results.

\section{Conclusions}

We found that CKD was independently associated with recurrent stroke, cardiovascular events or all-cause mortality independent of age, sex and the presence of cerebral SVD in patients with RSSI. There were robust relationships between WMH, microbleeds and the presence of CKD.

\section{Acknowledgments}

We are grateful to Naoko Akazawa, Tomoko Yunoki and Hiroko Ohta for their secretarial assistance. This work was supported in part by a research grant to N. Saji (Grants-in-Aid for Scientific Research No. 26870765) from the Japan Society for the Promotion of Science. 
Saji et al.: Chronic Kidney Disease Is an Independent Predictor of Adverse Clinical

Outcomes in Patients with Recent Small Subcortical Infarcts

\section{References}

1 Nakayama M, Metoki H, Terawaki H, Ohkubo T, Kikuya M, Sato T, Nakayama K, Asayama K, Inoue R, Hashimoto J, Totsune $\mathrm{K}$, Hoshi H, Ito S, Imai Y: Kidney dysfunction as a risk factor for first symptomatic stroke events in a general Japanese population: the Ohasama Study. Nephrol Dial Transplant 2007;22:1910-1915.

$\longrightarrow 2$ Miwa K, Tanaka M, Okazaki S, Furukado S, Yagita Y, Sakaguchi M, Mochizuki H, Kitagawa K: Chronic kidney disease is associated with dementia independent of cerebral small-vessel disease. Neurology 2014;82:10511057.

-3 Chinda J, Nakagawa N, Kabara M, Matsuki M, Endo H, Saito T, Sawada J, Katayama T, Sato N, Hasebe N: Impact of decreased estimated glomerular filtration rate on Japanese acute stroke and its subtype. Intern Med 2012; 51:1661-1666.

4 Yahalom G, Schwartz R, Schwammenthal Y, Merzeliak O, Toashi M, Orion D, Sela BA, Tanne D: Chronic kidney disease and clinical outcome in patients with acute stroke. Stroke 2009;40:1296-1303.

5 Kumai Y, Kamouchi M, Hata J, Ago T, Kitayama J, Nakane H, Sugimori H, Kitazono T; FSR Investigators: Proteinuria and clinical outcomes after ischemic stroke. Neurology 2012;78:1909-1915.

-6 Tsagalis G, Akrivos T, Alevizaki M, Manios E, Stamatellopoulos K, Laggouranis A, Vemmos KN: Renal dysfunction in acute stroke: an independent predictor of long-term all combined vascular events and overall mortality. Nephrol Dial Transplant 2009;24:194-200.

7 Oksala NK, Salonen T, Strandberg T, Oksala A, Pohjasvaara T, Kaste M, Karhunen PJ, Erkinjuntti T: Cerebral small vessel disease and kidney function predict long-term survival in patients with acute stroke. Stroke 2010; 41:1914-1920.

-8 Ito S, Nagasawa T, Abe M, Mori T: Strain vessel hypothesis: a viewpoint for linkage of albuminuria and cerebrocardiovascular risk. Hypertens Res 2009;32:115-121.

-9 Cho AH, Lee SB, Han SJ, Shon YM, Yang DW, Kim BS: Impaired kidney function and cerebral microbleeds in patients with acute ischemic stroke. Neurology 2009;73:1645-1648.

10 Khatri M, Wright CB, Nickolas TL, Yoshita M, Paik MC, Kranwinkel G, Sacco RL, DeCarli C: Chronic kidney disease is associated with white matter hyperintensity volume: the Northern Manhattan Study (NOMAS). Stroke 2007;38:3121-3126.

11 Ryu WS, Lee SH, Kim CK, Kim BJ, Yoon BW: The relation between chronic kidney disease and cerebral microbleeds: difference between patients with and without diabetes. Int J Stroke 2012;7:551-557.

12 Otani H, Kikuya M, Hara A, Terata S, Ohkubo T, Kondo T, Hirose T, Obara T, Metoki H, Inoue R, Asayama K, Kanno A, Terawaki H, Nakayama M, Totsune K, Hoshi H, Satoh H, Izumi S, Imai Y: Association of kidney dysfunction with silent lacunar infarcts and white matter hyperintensity in the general population: the Ohasama Study. Cerebrovasc Dis 2010;30:43-50.

13 Ikram MA, Vernooij MW, Hofman A, Niessen WJ, van der Lugt A, Breteler MM: Kidney function is related to cerebral small vessel disease. Stroke 2008;39:55-61.

14 Wardlaw JM, Smith EE, Biessels GJ, et al; Standards for Reporting Vascular Changes on Neuroimaging (STRIVE v1): Neuroimaging standards for research into small vessel disease and its contribution to ageing and neurodegeneration. Lancet Neurol 2013;12:822-838.

-15 Ovbiagele B, Wing JJ, Menon RS, Burgess RE, Gibbons MC, Sobotka I, German L, Shara NM, Fernandez S, JayamTrouth A, Edwards DF, Kidwell CS: Association of chronic kidney disease with cerebral microbleeds in patients with primary intracerebral hemorrhage. Stroke 2013;44:2409-2413.

16 Hashimoto J, Aikawa T, Imai Y: Large artery stiffening as a link between cerebral lacunar infarction and renal albuminuria. Am J Hypertens 2008;21:1304-1309.

-17 Adams HP Jr, Bendixen BH, Kappelle LJ, Biller J, Love BB, Gordon DL, Marsh EE 3rd: Classification of subtype of acute ischemic stroke. Definitions for use in a multicenter clinical trial. TOAST. Trial of Org 10172 in Acute Stroke Treatment. Stroke 1993;24:35-41.

-18 Saji N, Shimizu H, Kawarai T, Tadano M, Kita Y, Yokono K: Clinical features of a first-ever lacunar infarction in Japanese patients: poor outcome in females. J Stroke Cerebrovasc Dis 2011;20:231-235.

19 Saji N, Kimura K, Kawarai T, Shimizu H, Kita Y: Arterial stiffness and progressive neurological deficit in patients with acute deep subcortical infarction. Stroke 2012;43:3088-3090.

20 Saji N, Kimura K, Shimizu H, Kita Y: Association between silent brain infarct and arterial stiffness indicated by brachial-ankle pulse wave velocity. Intern Med 2012;51:1003-1008.

-21 Saji N, Kimura K, Shimizu H, Kita Y: Silent brain infarct is independently associated with arterial stiffness indicated by cardio-ankle vascular index (CAVI). Hypertens Res 2012;35:756-760.

22 Saji N, Shimizu H, Kawarai T, Tadano M, Kita Y, Yokono K: Increased brachial-ankle pulse wave velocity is independently associated with white matter hyperintensities. Neuroepidemiology 2011;36:252-257.

-23 Ovbiagele B, Liebeskind DS, Pineda S, Saver JL: Strong independent correlation of proteinuria with cerebral microbleeds in patients with stroke and transient ischemic attack. Arch Neurol 2010;67:45-50.

24 Wardlaw JM: Blood-brain barrier and cerebral small vessel disease. J Neurol Sci 2010;299:66-71.

25 Vlachopoulos C, Aznaouridis K, Terentes-Printzios D, Ioakeimidis N, Stefanadis C: Prediction of cardiovascular events and all-cause mortality with brachial-ankle elasticity index: a systematic review and meta-analysis. Hypertension 2012;60:556-562.

26 Ninomiya T, Kojima I, Doi Y, Fukuhara M, Hirakawa Y, Hata J, Kitazono T, Kiyohara Y: Brachial-ankle pulse wave velocity predicts the development of cardiovascular disease in a general Japanese population: the Hisayama Study. J Hypertens 2013;31:477-483, discussion 483. 